\title{
BREAST CONSERVATIVE SURGERY. A SURGICAL PROCEDURE WHICH COMBINES ONCOLOGICAL SAFETY AND ESTHETIC EFFECT
}

\author{
Nicolae Bacalbasa ${ }^{1}$, Olivia Ionescu ${ }^{2}$, Irina Balescu ${ }^{3}$ \\ 1 "Carol Davila" University of Medicine and Pharmacy, Bucharest, Romania \\ 2 "Bucur" Maternity Hospital, Bucharest, Romania \\ 3 "Ponderas" Hospital, Bucharest, Romania
}

\begin{abstract}
In the last twenty years, the surgical treatment of breast cancer (BC) has known a substantial progress, evolving from radical mastectomy - a mutilating procedure commonly performed in until 1970s - to breast conservative surgery (BCS) which allows the removal of the entire breast parenchyma saving the skin envelope of the mammary gland, and sentinel lymph node biopsy that helped reducing the complication rate associated with radical axillary dissection. As it has been proved that BCS and radical mastectomy are associated with similar survival rates, BCS or "skin sparing mastectomy" is nowadays considered to be an oncologically safe procedure in locally advanced BC if the surgical resection criteria is respected: negative surgical margins of resection with an entire removal of all the malignant breast tissue and the maintain of aesthetic result.

Moreover, owing to the development of novel oncoplastic techniques and the fact that performing BCS allows the preservation of the skin and sometimes the nipple-areola complex (NAC), BCS techniques such as the skin - sparing mastectomy and nipple-areola complex mastectomy are currently considered mainstay treatment of early stage $\mathrm{BC}$ without compromising the oncological safety of mastectomy.
\end{abstract}

Keywords: breast cancer, conservative mastectomy

\begin{abstract}
Abbreviations:
$\mathrm{BC}=$ breast cancer;

$\mathrm{BCS}=$ breast conservative surgery;

NAC $=$ nipple-areola complex;

$\mathrm{SSM}=$ skin-sparing mastectomy;

\author{
NSM = nipple-sparing mastectomy; \\ DCIS = ductal carcinoma in situ; \\ $\mathrm{NAC}=$ nipple-areola complex;
}

\section{INTRODUCTION}

Breast cancer $(\mathrm{BC})$ is the most common female cancer almost one million women being diagnosed annually worldwide. However, in recent years BC has registered a low mortality rate mainly in developed, high-income countries (1).

New developments have been registered in the surgical management of $\mathrm{BC}$, the oncological safety of the surgical resection combined with the aesthetic result being the goals that any modern surgeon should achieve (2).

Successful implementation of the screening programs and the population's increased awareness of the gravity of the disease have led to a higher rate of early-stage $\mathrm{BC}$ detection, hence being more eas- ily to promote the use of BCS in the clinical practice. Generally, BCS techniques are performed for T1 and T2 BC tumors without forgetting to pay attention to the fact that a complete remove of the tumor as well as a satisfactory cosmetic result are mandatory (3). The former aspect - obtaining of negative surgical margins - is required in order to minimize the risk of the rate of ipsilateral breast recurrences, which, in case of appearance, are associated with a low breast cancer - specific survival rate, while the latter mentioned aspect - the cosmetic result - causes less physical deformity and reduces possible fears related to the treatment such as changes in body image, sexuality and treatmentrelated distress (4). 
Locally advanced $\mathrm{BC}$ patients generally benefit from neoadjuvant chemotherapy that reduces the volume of the tumor and makes possible performing afterwards BCS techniques. However, it is important to know that is imperiously to consider pathologic response after neoadjuvant chemotherapy. In case of evidence of a small tumor with reduced volume with peripheric small nodules - the so called "mosaic" pattern, a total mastectomy should be performed in the second step of the treatment as the incidence of local recurrence if BCS is applied is still relative high (5).

Other situations in which BCS are not indicated include (6):

- Multicentric disease;

- Inflammatory BC;

- Contraindications to perform radiotherapy after BCS: pregnancy, previous breast-conserving therapy, diseases of the connective tissue;

- Indication to contralateral prophylactic mastectomy;

- Predisposition to breast cancer such as presence of germline BRCA1 or BRCA2 mutation.

Scientific evidence has continuously demonstrated that BC targeted systemic therapies are efficient in BC women a fundamental statement that resulted in a change from aggressive surgery - Halsted radical mastectomy-to more conservative surgical procedures which allow the preservation of skin and/or the NAC followed by reconstructive techniques using either autologous tissue or implant-based techniques (7).

Freeman has been the first who initially reported the procedure of "subcutaneous mastectomy" in patients with benign breast tumors (8). In malignant tumors, the "skin-sparing mastectomy" has been described by Toth and Lappert in 1991 who achieved a complete removal of the tumor as well as preserving the native breast cancer which automatically has resulted in a good aesthetic result (9). As various studies have reported that the local recurrence rate of radical modified mastectomy (Madden) and BCS are similar, the skin-sparing mastectomy procedure is considered to be the gold standard option in early stage BC and in some situations of locally-advanced BC $(10,11)$. As a skinsparing mastectomy also implies the removal of the NAC, immediate or delayed techniques aimed at the achievement of a satisfactory nipple reconstruction have progressively developed. However a nip- ple-sparing mastectomy seems to be a valuable option with both therapeutic and prophylactic indications (12).

In this review our goal was to present the indications, advantages and oncologic safety of the two most common performed conservative mastectomies: skin-sparing mastectomy (SSM) and nipplesparing mastectomy (NSM).

\section{INDICATIONS TO CONSERVATIVE MASTECTOMY}

BCS is a valuable alternative to women with small-medium size breast diagnosed with unifocal $\mathrm{BC}$ located in the central or in the inner quadrant(s). After bringing to attention the possible risk of leaving residual disease, patients with small to medium size breast and moderate or no ptosis diagnosed with unifocal breast cancer located in the lower outer quadrant can be supposed to BCS (13). Locally-advanced $\mathrm{BC}$ patients can benefit $\mathrm{BCS}$ procedures only if a good pathologic pattern - either pathologic complete response or concentric shrinkage - are demonstrated to be obtained after systemic neoadjuvant chemotherapy with a significant reduction in the tumor volume (14). Among the criteria used in selecting candidates for BCS after neoadjuvant chemotherapy we mention (15):

- Residual tumor size $<5 \mathrm{~cm}$;

- No evidence of multicentric lesion;

- Absence of extensive intramammary lymphatic invasion/extensive microcalcification;

- Complete resolution of skin edema.

With regard to the risks associated with skinsparing mastectomy, it appears that women who suffer from obesity, diabetes or who smoke, as well as those who received adjuvant radiotherapy are at risk of developing infections or skin necrosis if they opt for a skin-sparing mastectomy (16).

The NSM is preferred if a prophylactic mastectomy (e.g. presence of BRCA 1 or BRCA 2 mutations) is required without forgetting to intraoperative assess the involvement of the nipple-areola complex (17).

Among the contraindication of the nipple-sparing mastectomy we mention (18):

- Tumors with centrally localized or close to the nipple-areola complex;

- Severe breast ptosis.

Risk of the nipple-sparing mastectomy refer to: loss of sensibility of the nipple, partial or total necrosis of the NAC. 


\section{ONCOLOGICAL SAFETY OF SKIN-SPARING MASTECTOMY}

Apart from the above mentioned inclusion criteria for skin-sparing mastectomy in patients with early-stage and non-invasive $\mathrm{BC}$, this technique can also be used with prophylactic intention in women at high risk of developing $\mathrm{BC}$ who choose to receive a prophylactic mastectomy. The technique implies a complete dissection above the superficial fascia and the removal all the malignant breast tissue leaving only the epidermis, dermis and a small volume of the subcutaneous fat (19).

Patani and coworkers showed that the local recurrence rate after SSM and non SSM are similar (20). Moreover, SSM seems to be an independent predictor of obtaining close or positive margins. However, after a deep analyzation of the skin flaps specimens some authors did not conclude that the recurrence rates after SSM and non SSM are similar (21). For example, Torresan et al (22) proved that skin flaps thicker than $5 \mathrm{~mm}$ contain residual disease while Ho et al (23) that the residual malignant tissue in the skin flaps specimens are usually placed at the level of the skin projection of the tumor.

Even though many studies proved that the local recurrence rate after nipple - or SSM are similar $(24,25)$, Rocco and coworkers (19) have reported a local recurrence rate between $0 \%$ and $7 \%$ in patients submitted to SSM compared to that of $10 \%$ after 20 years of follow-up in patients submitted to NSM. On the other hand, lower recurrence rate after NSM which range between $1 \%$ and $3 \%$ have also been reported in women diagnosed with ductal in situ carcinoma (DCIS) (26), while Carlson ad his coworkers (27) that only one patient of 539 presented with a local recurrence after 65 months of follow-up following SSM for DCIS. They demonstrated that the tumor size, nodal status and the presence of the lymphovascular invasion are the most important predictors of recurrence with local recurrence rates of $3 \%, 10 \%$ and $11 \%$ for $\mathrm{T} 1, \mathrm{~T} 2$ and $\mathrm{T} 3$ tumors respectively.

Apart from tumor size, stage and lymph node involvement, a poor differentiation of the tumor constitutes a risk factor of developing a recurrence, the rate reported from $\mathrm{HO}$ and coworkers (23) after 75 month of follow-up being $4.5 \%$ quite similar to the $5.6 \%$ reported rate from Spiegel and Butler (28) in 117 patients treated with SSM after 9.8 years of follow-up.

\section{ONCOLOGICAL-SAFETY OF NIPPLE-SPARING MASTECTOMY}

As regards the incidence of $\mathrm{BC}$ in the NAC, the reported results in the literature are contradictory. For example, Laronga et al. (29) proved that risk factors of NAC involvement are centrally localized breast tumors, multicentricity, and the presence of metastatic lymph nodes. The reported incidence of tumors in the NAC has been reported to be influenced by the pathological definition of the NAC involvement, the histopathologic result (e.g. DCIS) as well as the volume of the tumor, and the selection criteria of the patients who can receive NAC (23).

Other study conducted by Cense and coworkers (30) took into consideration the volume of the tumor, the distance between tumor and NAC and confirmation of positive LN and found almost $58 \%$ of the mastectomy specimens presented involvement of the NAC. In contradiction to these results, the study of Simmons (31) showed that only $0,9 \%$ of the mastectomy specimens had nipple/areola involvement.

The local recurrence rate after performing NSM has been reported to be between $0 \%$ and $24,1 \%$ these percentages depending on the time of followup, the characteristics of the women included in different studies as well as the pre-and intraoperatives procedures (e. g. preoperative breast magnetic resonance imaging, intraoperative radiotherapy) $(32,33)$.

\section{IMPORTANCE OF THE ESTHETIC EFFECT}

There are many incision methods which can be used to ease the mastectomy and the reconstruction, to preserve the NAC blood flow and to obtain good aesthetic results. However, necrosis is more frequently when periareolar incisions are used compared to the transareolar approach (23).

BCS are generally most frequently preferred as it has been shown that they are both oncologically safe and they confer better esthetic outcomes (23). With regard to the differences between SSM and NSM in terms of aesthetic results, Gerber and coworkers (34) showed that both after SSM and NSM the patients declared that the aesthetic outcome is good or excellent. $74 \%$ and $26 \%$ of the patients who underwent NSM have reported excellent respectively good cosmetic effect, while the results were excellent, good and fair in 59\%, 22\% and $20 \%$, respectively in women who received SSM. 
Other study regarding the patient satisfaction showed that women are more satisfied in terms of body image, sexuality, cosmetic results and mutilation feeling after NSM than after SSM (35). Hence, we can conclude that the preservation of the nippleareola complex offers the women the possibility of conserving body image.

\section{CONCLUSIONS}

Conservative mastectomies offer a better quality of life for women with breast cancer. The low rates

\section{REFERENCES}

1. American Cancer Society. Cancer Facts and Figures 2010. Atlanta, Ga: American Cancer Society; 2010.

2. Franceschini G., Martin Sanchez A., Di Leone A., Magno S., Moschella F., Accetta C., Masetti R. New trends in breast cancer surgery: a therapeutic approach increasingly efficacy and respectful of the patient. G Chir. 2015 Jul-Aug; 36(4):145-52.

3. Chirappapha P., Kongdan Y., Vassanasiri W., Ratchaworapong K., Sukarayothin T., Supsamutchai C., Klaiklern P., Leesombatpaiboon M., Hamza A., Zurrida S. Oncoplastic technique in breast conservative surgery for locally advanced breast cancer. Gland Surgery, Vol 3, No 1 February 2014. http://www.glandsurgery. org/article/view/3406/4272

4. Ganz P.A. Psychological and social aspects of breast cancer. Oncology (Williston Park). 2008 May; 22(6):642-6, 650; discussion $650,653$.

5. Fisher B., Anderson S., Bryant J., et al. Twenty-year followup of a randomized trial comparing total mastectomy, lumpectomy, and lumpectomy plus irradiation for the treatment of invasive breast cancer. N Engl J Med 2002; 347:1233-41

6. Domchek S.M., Friebel T.M., Singer C.F., et al. Association of risk-reducing surgery in BRCA1 or BRCA2 mutation carriers with cancer risk and mortality. JAMA 2010; 304:967-75.

7. Kroll S.S., Schusterman M.A., Tadjalli H.E., et al. Risk of recurrence after treatment of early breast cancer with skinsparing mastectomy. Ann Surg Oncol 1997; 4:193-7.

8. Freeman B.S. Subcutaneous mastectomy for benign breast lesions with immediate or delayed prosthetic replacement. Plast Reconstr Surg Transplant Bull 1962; 30:676-82.

9. Toth B.A., Lappert P. Modified skin incisions for mastectomy: the need for plastic surgical input in preoperative planning. Plast Reconstr Surg 1991; 87:1048-53.

10. Carlson G.W., Bostwick J. 3rd, Styblo T.M. et al. Skinsparing mastectomy. Oncologic and reconstructive considerations. Ann Surg 1997; 225:570-5; discussion 575-8.

11. Manning A.T., Sacchini V.S. Conservative mastectomies for breast cancer and risk-reducing surgery: the Memorial Sloan Kettering Cancer Center experience.

12. de Alcantara Filho P., Capko D., Barry J.M., et al. Nipplesparing mastectomy for breast cancer and risk-reducing surgery: the Memorial Sloan-Kettering Cancer Center experience. Ann Surg Oncol 2011; 18:3117-22.

13. Krumholz H.M. Informed consent to promote patient centered care. JAMA 2010; 303:1190-1.

14. Prabhu R., Godette K., Carlson G., et al. The impact of skin-sparing mastectomy with immediate reconstruction in patients with Stage III breast cancer treated with neoadjuvant chemotherapy and postmastectomy radiation. Int J Radiat Oncol Biol Phys 2012; 82:e587-93. of local recurrence, the 5-year survival rates of more than $95 \%$ as well as the satisfactory esthetic results are other proofs that BCS can are associated with good cosmesis and maintenance of woman body image without compromising the oncological safety of mastectomy.

15. Singletary S.E., McNeese M.D., Hortobagyi G.N. Feasibility of breast-conservation surgery after induction chemotherapy for locally advanced breast carcinoma. Cancer 1992; 69:2849-52

16. Spear S.L. Surgery of the Breast Principles and Art. Third Edition. New York: Wolters Kluwer/Lippincott Williams and Wilkins; 2011.

17. Atisha D.M., Comizio R.C., Telischak K.M., et al. Interval inset of TRAM flaps in immediate breast reconstruction: A technical refinement. Ann Plast Surg. 2010; 65(6):524-27

18. Rusby J.E., Smith B.L., Gui G.P. Nipple-sparing mastectomy. Br J Surg. 2010; 97(3):305-16

19. Rocco N., Catanuto G., Bruno Nava M. What is the evidence behind conservative mastectomies? Gland Surgery, Vol 4, No 6 December 2015: http://dx.doi.org/10.3978/j.issn.2227-684X.2015.04.19

20. Patani N., Mokbel K. Oncological and aesthetic considerations of skin-sparing mastectomy. Breast Cancer Res Treat. 2008; 111(3):391-403

21. Sotheran W.J., Rainsbury R.M. Skin-sparing mastectomy in the UK -A review of current practice. Ann R Coll Surg Engl 2004; 86:82-6

22. Torresan R.Z., DosSantos C.C., Brenelli H., et al. Residual glandular tissue after skin-sparing mastectomies. Breast J 2005; 11:374-5.

23. Ho C.M., Mak C.K., Lau Y., et al. Skin involvement in invasive breast carcinoma: safety of skin-sparing mastectomy. Ann Surg Oncol 2003; 10:102-7.

24. Greenway R.M., Schlossberg L., Dooley W.C. Fifteen-year series of skin-sparing mastectomy for stage 0 to 2 breast cancer. Am J Surg 2005; 190:918-22.

25. Foster R.D., Esserman L.J., Anthony J.P., et al. Skin-sparing mastectomy and immediate breast reconstruction: a prospective cohort study for the treatment of advanced stages of breast carcinoma. Ann Surg Oncol 2002; 9:462-6.

26. Godat L.N., Horton J.K., Shen P., et al. Recurrence after mastectomy for ductal carcinoma in situ. Am Surg 2009; 75:592-5.

27. Carlson G.W., Styblo T.M., Lyles R.H., et al. Local recurrence after skin-sparing mastectomy: tumor biology or surgical conservatism? Ann Surg Oncol 2003; 10:108-12.

28. Spiegel A.J., Butler C.E. Recurrence following treatment of ductal carcinoma in situ with skin-sparing mastectomy and immediate breast reconstruction. Plast Reconstr Surg 2003; 111:706-11.

29. Laronga C., Kemp B., Johnston D., et al. The incidence of occult nipple-areola complex involvement in breast cancer patients receiving a skin-sparing mastectomy. Ann Surg Oncol 1999; 6:609-13.

30. Cense H.A., Rutgers E.J., Lopes Cardozo M., et al. Nipplesparing mastectomy in breast cancer: a viable option? Eur J Surg Oncol 2001; 27:521-6

31. Simmons R.M., Brennan M., Christos P., et al. Analysis of nipple/ areolar involvement with mastectomy: can the areola be preserved? Ann Surg Oncol 2002; 9:165-8.

32. Petit J.Y., Veronesi U., Orecchia R., et al. Nipple sparing mastectomy with nipple areola intraoperative radiotherapy: One 
thousand and one cases of a five years experience at the European institute of oncology of Milan (EIO). Breast Cancer Res Treat 2009; 117:333-8.

33. Petit J.Y., Veronesi U., Orecchia R., et al. Risk factors associated with recurrence after nipple-sparing mastectomy for invasive and intraepithelial neoplasia. Ann Oncol 2012; 23:2053-8

34. Gerber B., Krause A., Dieterich M., et al. The oncological safety of skin sparing mastectomy with conservation of the nipple-areola complex and autologous reconstruction: An extended follow-up study. Ann Surg 2009; 249:461-8.

35. Didier F., Radice D., Gandini S., et al. Does nipple preservation in mastectomy improve satisfaction with cosmetic results, psychological adjustment, body image and sexuality? Breast Cancer Res Treat 2009; 118:623-33. 